\title{
EN TORNO AL ANÁLISIS TÁCTICO DE LAS FORTIFICACIONES IBÉRICAS. ALGUNOS PUNTOS DE VISTA ALTERNATIVOS*
}

POR

\author{
FERNANDO QUESADA SANZ
}

\section{RESUMEN - ABSTRACT}

Los Iberos no emplearon maquinaria de asedio, ni en general guerra de asedio, en sus conflictos anteriores a la llegada de Amilcar Barca en 237 a.C. Sólo en zonas muy concretas (como Cataluña o Alicante) hay fortificaciones que muestran un limitado conocimiento de la poliorcética avanzada mediterránea, mientras que en la mayoría de los casos las fortificaciones no están diseñadas para resistir un asedio formal.

The Iberians, in their wars before the arrival of Amilcar Barca in $237 \mathrm{BC}$, did not use artillery or siege machines, or even practised formal siege warfare. Only in certain areas (such as Catalonia or Alicante) there are fortifications that show a limited knowledge of the advanced techniques of Mediterranean siege tactics. In the majority of cases, however, town walls were not designed to withstand a formal siege.

\section{PALABRAS CLAVE - KEY WORDS}

Cultura Ibérica. Fortificaciones. Armas. Guerra y sociedad.

Iberian Culture. Fortifications. Weapons. Warfare and Society.

\section{INTRODUCCIÓN}

Pretendemos en estas páginas realizar algunas reflexiones al hilo del artículo que, sobre el 'análisis táctico de las fortificaciones ibéricas' publicó en el número anterior de esta misma revista nuestro colega y buen amigo Francisco Gracia (Gracia, 2000). Dicho trabajo no era un estudio aislado, sino que es lógica evolución de otras investigaciones ya realizadas por el mismo autor sobre tema similar, que cita en su artículo, y que por razones de brevedad no vamos a repetir aquí.

La principal aportación de dicho estudio es que sostiene que las fortificaciones ibéricas reflejan incuestionablemente en algunos trazados defensivos, y para el periodo 500-250 a. C., el empleo de soluciones técnicas que implican un conocimiento de las máquinas de asedio propias del mundo greco-púnico (p. 157, p. 141). Igualmente, que desde en torno al 250 a. C., se aprecia más generalizadamente un elevado grado de desarrollo y elaboración, hasta el punto de que el empleo de las obras de Eneas y Filón de Bizancio resulta apropiado para su análisis (Gracia, 2000: 156-157).

\footnotetext{
* Estudio realizado dentro del proyecto DGICYT PB97/0057.
} 
Parte el trabajo del hecho de que el episodio mejor conocido de la poliorcética en Iberia en época prerromana es el asedio de Sagunto por Aníbal en 219 a. C.; y parte de la idea de que la narración de Livio muestra una elaboración de las técnicas defensivas, y un conocimiento de las máquinas de asedio por parte de los de Arse, que a su vez implican unas profundas raices del conocimiento poliorcético de los iberos, que se podrían remontar hasta fines del s. V a. C. (Gracia, 2000: 134). Esta propuesta viene necesariamente acompañada de su complemento: que el caso de Sagunto no es excepcional, sino extrapolable al resto de los iberos (ibidem p. 155).

Sin embargo, creemos que es posible sostener algunos puntos de vista complementarios e incluso divergentes de las opiniones de F. Gracia, que conviene explicitar pues sólo desde el debate se obtendrán conclusiones provechosas.

Nuestras opiniones alternativas pueden, a efectos de una mejor descripción y un más ordenado análisis, dividirse en tres grupos: planteamientos metodológicos, cuestiones generales y cuestiones de detalle.

\section{LAS FUENTES}

En primer lugar, hay una cuestión de base que debe discutirse: si las fuentes literarias que F. Gracia emplea con profusión pueden o no aplicarse en la forma en que él lo hace al estudio de las fortificaciones ibéricas.

En primer lugar, no tenemos nada - muy al contrario - en contra de que se utilicen fuentes muy tardías como Vegecio o Vitrubio, que en efecto beben de un conocimiento muy anterior, para identificar los puntos clave que, para un experto de la Antigüedad, debía reunir una fortificación (Gracia, 2000: 133). Nos parece metodológicamente valiente pero aceptable. Nada mejor que recurrir a los criterios antiguos para definir lo que un antiguo buscaba con su construcción defensiva. Hasta aquí nada en contra, siempre y cuando no se distorsione este principio metodológico para luego rellenar huecos en la documentación relevante, que ha de ser la propiamente ibérica.

En segundo lugar, el Dr. Gracia hace un breve pero jugoso análisis introductorio sobre las diferentes fuentes que hablan sobre Iberia (sobre todo Livio y Polibio pero también Silio Itálico y otros). Aquí empezamos a manifestar discrepancias: mientras que F. Gracia reconoce las dificultades que plantea Silio Itálico como fuente histórica, pero lo considera válido, nosotros hemos considerado desde hace años (Quesada 1997:e.g. 334) que sustancialmente su obra es inutilizable como fuente histórica. Silio Itálico (c. 26-102 d. C.) quería ser un nuevo Virgilio, y su obra una nueva Eneida de dimensiones mitológicas. En este contexto, los vascones y cántabros que incluye en los ejércitos de Aníbal de Cannas, o las descripciones del armamento regalado por las Oceani gentes (2, $395 \mathrm{ss}$.) al jefe cartaginés bastan para descalificarlo como fuente histórica minimamente fiable, y así se sostiene en la crítica histórica moderna. Ahora bien, teniendo en cuenta la importancia que la aceptación o no de algunas descripciones de Silio itálico (como su monstruosa falarica en Pun. 1,350 ss. que a nuestro juicio es una exageración poética a partir de la falarica real), la diferente valoración de la fiabilidad de Silio Itálico puede tener bastantes consecuencias.

En tercer lugar, hay algo a nuestro modo de ver inquietante en el uso que de las fuentes se hace a veces en el artículo que ha originado estas reflexiones, y es la extrapolación siglos atrás en el tiempo de acontecimientos narrados en una época que fue, por definción, excepcional. A partir del 237 a. C. el mundo ibérico se vió envuelto en la Guerra Mundial de la época, sus tropas fueron masivamente empleadas como aliadas o auxiliares de los ejércitos de corte helenístico enfrentados en tierra hispana, y la guerra se prolongó años enteros. Todo esto era nuevo en la experiencia ibérica. Por ello, nos removemos inquietos cuando se se 
aplican citas de Tito Livio (sobre los años 218-206) a la discusión sobre si desde el s. V a. C. había asedios en el mundo ibérico (Gracia, 2000: 136)ํ. Lo mismo ocurre con la extrapolación hacia atrás de situaciones tácticas romanas o cartaginesas descritas por las fuentes para el final del s. III a. C. (ibidem pp. 135 ss. passim). Creemos que, para tratar de probar la existencia de una poliorcética elaborada en los ss. V-III a. C. hubiera sido metodológicamente más adecuado renunciar por completo a esos textos tardíos y a menudo referidos a romanos o cartagineses, y ceñirse a la evidencia arqueológica, máxime cuando incluso esos textos no hablan tampoco de asedios por parte de iberos a otros iberos. Si la arqueología resulta convincente aquí, no nos hará falta el recurso a 'arrastrar' las fuentes dos o tres siglos hacia atrás en el tiempo, y a circunstancias por completo diferentes. Y si no lo es, las fuentes literarias no pueden cubrir el hueco por mucho que lo intentemos.

\section{¿CONOCÍAN LOS IBEROS LAS MÁQUINAS DE ASEDIO?}

Creemos que hay un cierto non sequitur en la base de la argumentación de Gracia. Cómo en el asedio del 219 los saguntinos parecen según Livio conocer bien las técnicas poliorcéticas mediterráneas, este conocimiento debe remontarse a un periodo anterior, que se lleva de manera algo arbitraria, a nuestro juicio, hasta el s. V a. C. (Gracia, 2000: 134). Una vez que estamos en ese momento, es necesario identificar el origen de ese conocimiento, que se remite para Gracia al: 'contacto con los comerciantes y colonos semitas y/o foceos', 'por la observación del sistema defensivo de Emporion' y por 'la presencia de mercenarios ibéricos en el Mediterráneo central y oriental' (ibidem p. 134).

Ahora bien, consideramos irrelevante el non sequitur citado porque hay fortificaciones del s. V con rasgos elaborados, y aceptamos pues el razonamiento. La pregunta que se plantea entonces aquí es pues doble: $a$. ¿conocían de verdad los saguntinos del 219 a. C. lo más avanzado de la poliorcética helenística? y $b$. ¿de verdad ese conocimiento es extrapolable al conjunto del mundo ibérico y hasta el s. V a. C.?. El punto $(a)$ es indemostrable, basado como está sólo en pocas fuentes literarias de las que sólo Livio es en realidad sólido. Con todo, vamos a coincidir con Gracia en que por la narración de las fuentes se deduce que en efecto los saguntinos 'estaban muy puestos'. Pero, ¿qué pasa con el punto $(b)$ ?.

Gracia reconoce paladinamente (p. 135) que no hay fuentes sobre asedios en Iberia antes de Aníbal. Sin embargo mantiene (ibidem) que la sabiduría heredada de los saguntinos, y la propia estructura defensiva de las fortificaciones ibéricas, denuncian que se dieron asedios, y con máquinas de guerra, antes de esa fecha ${ }^{2}$. Puesto que la primera cuestión es indemostrable pero la hemos aceptado para poder seguir adelante, y puesto que en todo caso no podemos saber hasta qué fecha se remontaría esa herencia poliorcética, parece razonable centrarse en la evidencia arqueológica. Pero Gracia no se concentra en ello, y no a fondo, hasta mucho más adelante en su discurso (p. 150 ss.). Si pudiera demostrar satisfactoriamente que desde el s. V a. C. las fortificaciones iberas estaban en buena parte construidas teniendo en cuenta técnicas de asedio muy elaboradas que incluyeran el empleo de máquinas de guerra, su hipótesis quedaría probada.

Es sin embargo aquí donde se concentran la mayoría de nuestras discrepancias de alcance. Porque en efecto el profesor Gracia puede presentar varios casos de fortificaciones ver-

\footnotetext{
1 Además, las citas son reveladoras: siempre se habla de asaltos violentos y no de asedios.

2 Un tercer argumento, basado en las noticias de las fuentes para las guerras del periodo post -218, a saber, que los Iberos hacían salidas, perseguían a enemigos en retirada tras un asedio fallido, o que acopiaban víveres, nos parece directamente desechable. En primer lugar, no prueba nada para el periodo anterior al 218, puesto que las respuestas tácticas que se citan son obvias y no requieren una experiencia previa en asedios prolongados. En segundo lugar, en todos los casos se trata de asedios por parte de extranjeros a núcleos ibéricos, y no de asedios iberos.
} 
daderamente complejas, con torres avanzadas, proteichisma, etc. (p. 137 y sobre todo 150 ss.). Sin embargo, esos ejemplos se concentran en unos pocos - muy pocos- yacimientos, sobre todo del área catalana y en particular de la periferia de Emporion. La modulación encontrada en el Puig de Sant Andreu de Ullastret o en la Picola de Alicante es excepcional y no la norma en las fortificaciones ibéricas (vease el completo catálogo de Moret, 1996).

Pero el Profesor Gracia va además mucho más allá: según su propuesta, los Iberos conocerían de antiguo no sólo la técnica de defensa contra máquinas de asedio (que, concedemos, podrían quizá haber copiado de modelos de fortificación sin comprenderlos bien), sino también las propias máquinas (Gracia, 2000:141). Para ello, sin embargo, la argumentación que se emplea es a nuestro juicio débil, por la sencilla razón de que no hay datos para sostener esa hipótesis, y sí varios para desecharla. Veamos: Gracia admite que no hay restos arqueológicos de máquinas antes de mediados del s. I a. C., pero sostiene que muchas fortificaciones demuestran la existencia de las mismas por su disposición. Cita algunas fortificaciones catalanas (Ullastret, Castellet de Banyoles, Turó del Montgrós, Alorda Park), además de La Serreta de Alcoy y Capote en Badajoz 33 (p. 141, de nuevo en p. 142 y p. 149).

Ahora bien, estos yacimientos, y la mayoría del resto de los iberos, a excepción de Montgrós, Ullastret y Banyoles, no muestran estructuras de tipo helénico o helenístico que impliquen verdaderos conocimientos poliorcéticos, y si rasgos que indican su desconocimento. Por ejemplo, la separación entre torres de Ullastret reflejaría un mal conocimiento del alcance de las armas arrojadizas, con un exceso de precaución, pues las torres se encuentran el doble de próximas entre sí de lo que sería necesario según los propios cálculos que acepta Gracia (p. 140). El propio análisis de la estructura de Banyoles, la más fiel en apariencia a los modelos helenísticos, demuestra que los iberos copiaron 'mal' sus modelos, despojándoles de buena parte de la potencialidad defensiva de los modelos originales que imitaban (Moret, 1998: 89; 1996: 217). Creemos que Moret ha mostrado claramente que la disposición de torres poligonales en casos como Tivissa no mejora la capacidad defensiva de la obra, ni implica la utilización de catapultas por parte defensores o atacantes. Y eso ocurre con uno de los ejemplos supuestamente más avanzados de las fortificaciones ibéricas. De igual forma si, como quiere Gracia, el alzado de adobe o tapial de la mayoría de las fortificaciones ibéricas se hubiera realizado para disminuir el efecto de proyectiles de asedio (pp. 150-151), las fortificaciones de época tartésica también tendrían la misma causa... siglos antes de la aparición en el Mediterráneo Oriental de las primeras máquinas de proyectiles (e.g. Ruiz Rodríguez, Molinos, Choclán, 1991: 114 ss.). El principio de Occam nos indica que el zócalo de piedra con alzado de barro es el sistema que mejor combina solidez y economía de esfuerzo en la construcción de una muralla de época preindustrial, y por tanto lo que cabe esperar haya catapultas o no.

Así pues, lo que muchas de estas fortificaciones parecen mostrar es precisamente que los iberos en ocasiones copiaron mal, sin entenderlos cabalmente, modelos diseñados para una guerra con armas de asedio... precisamente porque ellos no empleaban dichas máquinas. Resulta pues que la aplicación de la navaja de Occam resulta en una hipótesis mucho más acorde con los datos disponibles. Por si fuera poco, en el amplísmo panorama de las fortificaciones ibéricas (Moret 1996), las fortificaciones con la sofisticación de la media docena de ejemplos repetidamente citados por Gracia son poquísimas. Bien al contrario, en la inmensa mayoría de los casos encontramos lienzos formados con las traseras de las casas, alguna torre cuadrada para proteger la puerta y poco más. En conjunto (y no recurriendo a esa media docena de casos, sobre todo del ámbito catalán), el panorama de las fortificaciones de la Edad

3 Sin embargo, la fortificación de acceso a Capote está pendiente de excavación completa (la reconstrucción se basa en termografías), y el propio excavador habla de un conocimiento 'al menos nominal' de las técnicas defensivas helenísticas (Berrocal, 1994:34). En cuanto a la Serreta, no vemos en ella nada que implique un conocimiento de las máquinas de asedio por parte de sus constructores, ni siquiera en la estructura de la puerta (Olcina et alii. 1998:40-41). 
del Hierro es bastante más sencillo, con técnicas poco complejas que no reflejan temor a un asedio en regla, sino más bien a un asalto repentino (que es lo único que, recordemos, testimonian las fuentes literarias en raras ocasiones).

Por otro lado, y dada la escasez de argumentos arqueológicos, F. Gracia ha de recurrir a otros elementos para reforzar su argumentación, empleando para ello fuentes literarias extrapoladas hacia atrás. Por ello, en un intento de mostrar el conocimiento de las técnicas poliorcéticas por los iberos y antes de la Segunda Guerra Púnica introduce ejemplos como el asalto de Cartagena por Escipión (romanos contra una ciudad púnica, amurallada según cánones cartagineses y defendida por cartagineses, pág. 137, de nuevo en p. 140); la toma de Sagunto (los sitiadores son cartagineses, por tanto es obvio que emplean técnicas avanzadas; pp. 138139, 151-152, 154...); asaltos romanos en Masalia en el 48 a.C. (p. 139, p. 154...), o en la Ulterior en el 78 a. C. (p. 139). Del mismo modo, el enorme arsenal capturado por Escipión en Cartagena era púnico, no ibero, y por tanto las referencias (p. 140,141) en nada ayudan a demostrar que los iberos conocieran desde el $\mathrm{s}$. $\mathrm{V}$ a. $\mathrm{C}$. el empleo de las máquinas y su defensa contra ellas. Si eliminamos estos textos, que no son relevantes para la demostración que se persigue, la argumentación basada en la literatura queda convertida en un cascarón hueco. Los arietes, torres de asedio, máquinas y demás son siempre en las citas romanos o púnicos, no iberos.

Por otro lado, cuando se trata de presentar algún dato directo sobre la existencia de máquinas entre los iberos, sólo puede el Dr. Gracia (p.141) contar con la autoridad de Silio Itálico (una 'balista focea' adscrita a los defensores de Sagunto), cuya validez histórica hemos negado antes. En cuanto a las armas incendiarias citadas, de todas ellas solo la falarica tiene que ver con Hispania, pero el arma descrita por Livio no es sino una jabalina o pilum con una estopa prendida, y nada más (Quesada, 1997:334ss.). El caso de la ciudad de los Ausetanos (Livio, 21, 61, 10) no autoriza a nada más que a pensar en jabalinas o fuegos de fortuna, lo que en sí ya es bastante, pero no exige la presencia de máquinas ni mucho menos, que sin duda hubieran llamado la atención de los romanos.

Sólo en un caso hay una cita que parece pertinente, para F. Gracia tanto como para P. Moret (1996: 256-257). En el asalto romano a Orongis en 207 a. C., nos habla Gracia del 'fuego de barrera' de los defensores ibéricos (Gracia, 2000:139). Pero los 'proyectiles' que cita Livio $(28,3)$ son tela, no se especifica que sean dardos de máquina, sino jabalinas de todo tipo. Pero es que además Orongis está guarnecida y defendida por cartagineses (Livio, 28, 3, 9-11) quienes serían los principales defensores y los que lógicamente manejarían los garfios y horcas para rechazar las escalas. Nada nos autoriza a pensar que ni siquiera estos - por otro lado elementalísimos - ingenios defensivos fueran propios de los defensores iberos, - $\mathrm{y}$ en esto diferimos de Moret. Y no se mencionan otros más elaborados.

En conjunto, pues, creemos que ni la Arqueología ni las fuentes literarias nos autorizan a pensar que los iberos hubieran empleado la maquinaria de asedio propia del mundo helénico y helenístico. Por otro lado, tampoco vemos, salvo en casos muy concretos - que se reducen prácticamente al Puig de Sant Andreu de Ullastret y, si se quiere, a Sagunto - una técnica defensiva especialmente elaborada que responda a una necesidad de defensa pensada contra asedios formales con o sin máquinas. En resumen, toda la información disponible viene a resumirse en que Aníbal primero, y los romanos después, emplearon en Iberia técnicas de asedio y defensa elaboradas. Pero ningún texto ni evidencia arqueológica o iconográfica nos autoriza a trasladar esos datos al ámbito indígena, y menos aún antes del 237 a.C. Los escasos ejemplos de verdadera complejidad en la arquitectura defensiva se debieron a nuestro juicio más a imitaciones de modelos de prestigio orientales, helénicos o helenísticos, que a una verdadera necesidad ante una guerra de asedio que a nuestro juicio los iberos, simplemente, nunca practicaron. 


\section{MERCENARIOS, RUTAS, ARCOS Y HONDAS}

Hay también algunas cuestiones de detalle que merece la pena comentar. Una de ellas es la referente al posible papel de los mercenarios iberos en Sicilia como fuente de conocimiento poliorcético (p. 134-135). A ese tema hemos dedicado un extenso trabajo al que remitimos (Quesada, 1994). En esencia, creemos que la inmensa mayoría de los mercenarios que partieron hacia Sicilia entre los siglos V-III a. C. rara vez regresaron a sus tierras de origen, sino que murieron, permanecieron décadas en servicio o acabaron estableciéndose en tierras extranjeras. Hay abundante información literaria que documenta todo esto, pero remitimos para detalles al trabajo citado. Por tanto, estos mercenarios, que ademas no se 'helenizaban', no pudieron actuar como agentes de aculturación. Otro es el caso de los jefes que sí podían regresar con cierta frecuencia, enriquecidos y con prestigio, y que, coincidimos en ello con Gracia, pudieron actuar como agentes transmisores (no necesariamente los únicos) de una idea de fortificación entendida sobre todo como elemento de prestigio aupado sobre una básica funcionalidad defensiva. En nuestra opinión, sin embargo, esta transmisión pudo también ser responsable de la mala intepretación que de soluciones arquitectónicas pensadas para ámbitos militares mucho más exigentes, hicieron a veces los maestros de obra ibéricos.

En segundo lugar, nos parece atractiva la hipótesis planteada de que la experiencia de Sagunto en 219 a. C. llevara a Anibal a evitar cuidadosamente los poblados fortificados en su camino hacia el norte del año siguiente (p. 156).

Hay además otra cuestión concreta que no queremos tampoco dejar de mencionar, aunque no pretendemos a volver a repetir una argumentación que creemos haber desarrollado con suficiente capacidad de convicción en otros lugares. Nos referimos a la cuestión del empleo del arco y de la honda en el mundo ibérico.

Recordemos en primer lugar que no hay en toda la iconografía ibérica o celtibérica ninguna representación de arcos, flechas u hondas, pese a lo cual no hemos podido convencer a F. Gracia, quien afirma de nuevo que en la cerámica de Liria hay o puede haber un arco, pese a que nos parecía ya demostrado que se trata de un escudo visto en sección, con su empuñadura, umbo y su superficie exterior de piel, como en otros varios ejemplos del mundo ibérico y celtibérico (Gracia, 2000: 147; Quesada, 1997: 465 ss. y sobre todo la imagen de la pieza en Quesada, 1997: 467, Fig. 281, con paralelos allí citados).

Por demás, nuestra postura al respecto es clara (Quesada, 1997:435-480; 1989): los iberos conocieron el arco y la honda, aunque esta última sin proyectiles de plomo hasta siglos después de su aparición en el mediterráneo Oriental, probablemente hasta el s. II a.C. Las puntas de flecha y glandes de honda están desde c. 500 y hasta c. 100 a.C. prácticamente ausentes en el registro arqueológico funerario de Iberia (los ejemplares de cada tipo se pueden contar con los dedos de las manos, frente a los literalmente miles de lanzas/jabalinas o los centenares de espadas o escudos). En cuanto a los hábitats, en el intervalo 500-100 a. C., los muchos centenares de poblados ibéricos excavados han proporcionado un número muy bajo (menos de un centenar) de puntas de flecha, ridículo incluso en comparación con los miles de puntas de flecha de tipo semita halladas en las areas punicizadas de Andalucía Occidental. Estas puntas (insistimos que muy escasas comparativamente, aunque vayan apareciendo más) testimonian el conocimiento del arco y su empleo en la caza, pero no implican en absoluto su importancia en la guerra, sino más bien al contrario.

En efecto, la ausencia de estos tipos de arma en un registro tan simbólico y significativo como el funerario, donde sin embargo abundan los demás tipos de armas, unida a la ausencia completa de representaciones en la iconografía, y a la casi completa ausencia de menciones en las fuentes literarias, sólo puede significar una cosa: que entre el s. V y finales del II a. C. las armas arrojadizas propulsadas fueron despreciadas, como en muchas otras regiones y épocas, y consideradas afeminadas para su uso en la guerra por los hombres libres. Esto no 
impide que fueran conocidas y probablemente empleadas en la caza por los plebeyos, e incluso - aunque lo dudamos mucho - en la guerra por una infantería ligera de la que las fuentes no nos dicen casi absolutamente nada (honderos baleares aparte).

Sólo antes de principios del s. V a. C. (en una situación cultural diferente) y desde fines del s. II a.C. vuelven a ser frecuentes las puntas de flecha y los glandes en contextos de poblado, y muy ocasionalmente en tumbas. Así pues, sólo cuando romanos y púnicos batallaron en suelo hispano volvemos a encontrar abundancia de estas puntas y glandes, pero el contexto bélico es ya completamente distinto al original, e implica la llegada de nuevas unidades y tácticas ajenas a la guerra tal y como era tradicional en el mundo ibérico.

Veamos la evidencia que recoge F. Gracia para argumentar en contrario, esto es, que tanto la honda como el arco fueron abundantemente empleados por los iberos en la guerra de asedio desde antes del 237 a.C. En realidad, parte el autor de una consideración a priori: estas armas son especialmente útiles en dicho contexto bélico, por tanto, y como se conocían, debieron ser muy usadas (p. 143).

Para la honda, y aparte de nuevo del discutible Silio Itálico para el asedio de Sagunto (ibidem p. 143), no se puede aportar casi nada más, puesto que un interesante resumen histórico de la honda, desde Asiria a la Biblia, no aporta contenido a la argumentación de fondo. En absoluto puede decirse (p. 143) que las fuentes documenten ampliamente el empleo de la honda por los iberos (si entendemos, claro está, que los baleares no son iberos). Justo al contrario: un análisis cuidadoso de las mismas demuestra (Quesada, 1997: 479-480 para los detalles) que las pocas que hay son de época romana, y asociadas el ejército romano, salvo unicamente Estrabón 4, 15). Las referencias a los contingentes baleares reclutados por Aníbal no implican en absoluto que los iberos de los ss. V-III a. C. también los contrataran. No hay el más mínimo indicio al respecto, e incluso si tal cosa hubiera ocurrido (por ejemplo, entre los saguntinos, para los que no hay más indicio que algunos glandes de plomo de época romana), ello sólo vendría a confirmar que, entre los iberos propiamente dichos, la honda era un arma ocasional en el mejor de los casos. El análisis arqueológico que nosotros hemos realizado muestra que los glandes de plomo aparecen ya desde fines del siglo III y sobre todo en el I a. C., en la época crepuscular del mundo ibérico (Quesada, 1997: 477 ss.). Entonces sí se usaron, pero estamos hablando de unas circunstancias ya totalmente diferentes a las de los siglos V y IV a. C.

Sobre el arco, nada tenemos en absoluto, menos aún que para la honda. Tiene la amabilidad el profesor Gracia de citar nuestra opinión, resumida en párrafos anteriores, y recoger algunas de los datos sobre el desprecio caballeresco del arco en el Mediterráneo, y viene a plantear una solución de compromiso: el arco no sería apenas empleado en la batalla campal, pero sí en los asedios (pp. 145-146). Algo así opinababa también P. Moret en 1996 (Moret, 1996: 259). Nosotros en cambio no acabamos de verlo así. Como sigue sin haber evidencia textual directa, propone el prof. Gracia incluir las flechas entre los dardos que en Sagunto cita Livio (tela, missilia). Una razón es logística: los saguntinos, si constantemente hubieran arrojado jabalinas, se hubieran quedado sin suministro. No hay sin embargo razón alguna para esta propuesta: Livio es muy específico con términos como falarica o tragula (Quesada, 1997: 331 ss. para el campo semántico de estas armas), y cuando quiere usa sagittae sin problemas. Missilia solo en rarísimas ocasiones se emplea por sagittae (Hor. Carm. 3, 6, 16). En el mejor de los casos, pues, se trata de un argumento forzado: hubo de haber flechas por su utilidad y como hay razones logísticas que impedirían la abundancia masiva de jabalinas, ergo el vocablo 'dardos' en Livio ha forzosamente de incluir 'flechas'. No podemos compartir esa línea argumental.

En cuanto a la escasísima evidencia arqueológica para el arco, ha sido ya recogida y comentada por nosotros, aunque naturalmente se han producido incorporaciones a nuestro 'Catá- 
logo' de 1997 que no alteran el cuadro global en absoluto ${ }^{4}$. Insistimos: cien o más puntas de los ss. V-III a.C. no son realmente significativas nada en el cómputo global de armas conocidas.

F. Gracia también argumenta que el empleo por los romanos de arqueros en Hispania ${ }^{5}$ se debió a la necesidad de 'contrarrestar la profusión del empleo del arco entre sus enemigos cartagineses e iberos' (p. 147). No hay el más leve indicio positivo de ello: desde luego no hay dato alguno en las fuentes que lo apoye, y muy leves indicios podrían buscarse en la arqueología. Por un lado, las fuentes son mucho más tardías, referentes al 133 a.C.; por otro, ninguna fuente romana alude jamás al empleo de arqueros por los iberos, y dado que los romanos apenas empleaban el arco en la guerra en este periodo, si los iberos los hubieran tenido las fuentes lo reflejarían. Arqueológicamente, ya hemos aludido que sólo a partir del s. II hay cierta presencia de puntas de flecha en poblados, mientras que hasta ese momento son muy escasas y separadas entre sí, aunque la lista acumulada pueda parecer grande (Quesada, 1997:459 ss.).

\section{CONCLUSIÓN Y ALTERNATIVA}

En conclusión, pues, no creemos que los iberos emplearan entre los siglos V y III a. C. maquinaria de asedio, ni que recurrieran a ese tipo de guerra, ni que sus fortificaciones - salvo quizá en una docena de casos- reflejen un conocimiento alto de las técnicas poliorcéticas avanzadas del Mediterráneo. Tampoco creemos que emplearan abundantemente el arco y la honda desde el s. V a. C. en la defensa o ataque de fortificaciones, aunque quizá sí minoritaria y ocasionalmente. No negamos la introducción de técnicas más complejas desde circa 237 a. C., e incluso mucho antes en puntos muy concretos como el entorno de Ampurias, el del Bajo Ebro, Alicante o zonas de Andalucía.

Somos por otro lado conscientes de que las afirmaciones negativas son más fáciles que las constructivas, por lo que queremos al menos trazar aqui un brevísimo esbozo de nuestra visión alternativa del funcionamiento militar de las fortificaciones ibéricas, bien entendido que no es este lugar para una argumentación detallada, que es desde luego tema para otro trabajo ${ }^{6}$.

Así a nuestro juicio es posible construir un modelo alternativo para el empleo de las fortificaciones ibéricas que en conjunto resulta acorde con lo que sabemos sobre el armamento y tácticas del Ibérico Pleno, e incluso con la organización de la sociedad ibérica. Sostenemos que la defensa, tal y como era concebida por los iberos, era una defensa activa, basada en salir de los recintos fortificados y ofrecer batalla al enemigo que saqueaba los campos. Lo contrario, en este tipo de guerra (a la vez económica y de prestigio) hubiera supuesto dos derrotas graves: la pérdida de cosechas y ganados, y la de honor y prestigio (del jefe ante la comunidad, y de los hombres ante sus mujeres). Por eso los recintos fortificados sólo pueden, en la mayoría de los casos, detener asaltos por sorpresa o impedir la entrada de alima-

4 Bien por nuevos hallazgos o por piezas que se nos habían escapado por proceder de museos locales o contextos dudosos. El material de excavación no publicado todavia, obviamente, no puede ser tenido en cuenta. La fecha de un descubrimiento es la de su publicación, y no se puede pedir a nadie que conozca lo que no se ha publicado, pese a lo cual observamos con preocupación la creciente serie de afirmaciones de alcance que se realizan sobre material todavía inédito y que por tanto no pueden contrastarse.

5 El texto que se cita como referente a unos arqueros que llegarían a Hispania con Escipión en 207 a. C. (Livio 27, 38) no dice tal: los 3.000 arqueros y honderos van a Sicilia. La primera referencia hispana es de Apiano (Iberia 92) y Ps. Frontino $(4,7,27)$, quienes afiman que el otro Escipión, el Emiliano los utilizó, junto a honderos, mezclados con las centurias frente a Numancia (Quesada, 1997:468 y 480). Creemos que se han mezclado dos citas diferentes o que la redacción del párrafo lleva a engaño.

6 Presentado en marzo de 2001 en el marco del seminario Defensa y territorio en Hispania de los Escipiones a Augusto, celebrado en la Casa de Velazquez bajo la coordinación de F. Cadiou, D. Hourcade y A. Morillo, y en curso de publicación. 
ñas... no fueron concebidos para rechazar asedios formales que nunca vendrían, no sólo porque las fortificaciones ejercieran un efectivo papel militar disuasorio — también-, sino porque el atacante no tendría ningún interés en asediar la ciudad. Un asalto rápido o por sorpresa a una granja, bien; una entrada en tropel por una puerta abierta cuando se perseguía a un enemigo en huida, de acuerdo... pero un asedio prolongado carecería de sentido en la forma ibérica de entender la guerra... más valdría volver a saquear los campos y buscar la sorpresa la primavera siguiente.

Cuando cartagineses y romanos dieron un salto cualitativo en la forma de entender la actividad bélica, convirtiendo en un fin la toma -y subsiguiente arrasamiento en muchos casos- del núcleo fortificado, la única salida fue la resistencia a ultranza, pues la alternativa era una vida de esclavitud — que no de servidumbre, que podía ser tolerada - nunca o casi nunca contemplada antes de la llegada de las potencias civilizadoras.

FERNANDO QUESADA SANZ

Universidad Autónoma de Madrid

fernando.quesada@uam.es

\section{BIBLIOGRAFÍA}

BERROCAL RANGel, L. (1994): «Arqueología de las fortificaciones griegas (iii). Repercusiones entre los púnicos, Iberos y Celtas». Rev. de Arqueología 166, 24-35.

Gracia Alonso, F. (2000): «Análisis táctico de las fortificaciones ibéricas».Gladius 20, 131-170.

MoRET, P. (1996): Les fortifications ibériques. De la fin de l'Âge du Bronze à la conquête romaine. Madrid.

MORET, P. (1998): «Rostros de piedra. Sobre la racionalidad del proyecto arquitectónico de las fortificaciones urbanas ibéricas». Actas del Congreso Internacional, Los Iberos, príncipes de Occidente. Barcelona, pp. 83-92.

OlCiNA DOMÉNeCH, M. et alii (1998): «Nuevas aportaciones a la evolución de la ciudad ibérica: el ejemplo de La Serreta». Actas del Congreso Internacional, Los Iberos, príncipes de Occidente. Barcelona, pp. 35-46.

QuESADA SANZ, F. (1989): «La utilización del arco y las flechas en la Cultura Ibérica». Trabajos de Prehistoria 46, 161-201.

QuESADA SANZ, F. (1994): «Vías de contacto entre la Magna Grecia e Iberia: la cuestión del mercenariado». Arqueología de la Magna Grecia, Sicilia y la Península Ibérica. Córdoba, pp. 191-246.

QUESADA SANZ, F. (1997): El armamento ibérico. Estudio tipológico, geográfico, funcional, social y simbólico de las armas en la Cultura Ibérica (siglos VI-I a. C.). Monographies Instrumentum, 3. Montagnac.

Ruiz RodrígueZ, A.; Molinos, M.; ChOClán, C. (1991): «Fortificaciones ibéricas en la Alta Andalucía». Fortificacions. Simposi internacional d'arqueología ibèrica. Manresa, pp. 109-126. 